\title{
SUBJECT INDEX, VOL. 43, 2001
}

\author{
${ }^{10} \mathrm{Be}, 759-764$ \\ ${ }^{137} \mathrm{Cs}, 869-878$, 917-928 \\ ${ }^{13} \mathrm{C}, 555-560$ \\ ${ }^{14} \mathrm{C}$, (see also Radiocarbon) 263-270, 473-476, \\ 595-602, 751-758, 783-792, 809-820, 917-928, \\ 1057-1063 \\ ${ }^{14} \mathrm{C}$ Age, 555-560 \\ ${ }^{14} \mathrm{C}$ Apparent Age, 671-678 \\ ${ }^{14} \mathrm{C}$ Background, 169- 176 \\ ${ }^{14} \mathrm{C}$ Geophysics, 731-742 \\ ${ }^{210} \mathrm{~Pb}, 517-526,917-928$ \\ ${ }^{228} \mathrm{Ra}, 917-928$ \\ ${ }^{32} \mathrm{Si}, 909-916,917-928$ \\ $\Delta \mathrm{R}, 461-464,483-488$
}

Aboriginal Arrival in Australia, 1139-1146

Absolute Dating, 909-916, 917-928

Accelerator Mass Spectrometry (AMS), 83-86, 149-156, 163-168, 169- 176, 221-228, 229-238, 239-248, 283-292, 305-312, 313-320, 489-494,

703-710, 751-758, 821-830, 899-908, 1049-1056, 1057-1063, 1109-1114

Accuracy, 321-324

Acid-Base Wet Oxidatation (ABOX), 45-54, 239-248

Altai, 425-432

Amazon Region, 821-831

Amino Acids, 711-722

Antarctica, 751-758

Anthropogenic ${ }^{14} \mathrm{C}, 659-670,869-878$

Apatite, 249-254

Aquifer, 773-782

Arabian Sea, 229-238, 403-416, 417-424, 483-488, 965-976, 1049-1056, 1147-1154, 1155-1166,

1217-1222

Archeaology, 229-238, 403-416, 417-424, 965-976, 1049-1056, 1147-1154, 1115-1166

Arctic, 495-516

Arid-Semiarid Zone, 619-628

Art Dating, 1064-1076

Art History, 229-238

Artificial Neural Networks, 365-372

Asia, 1085-1109

Atlantic Ocean, 879-886

Atmospheric Radiocarbon, 339-345

Auramared System, 229-238

Australia, human occupation 1139-1146

Baden Culture, 1057-1063

Barents Sea, 843-856

Bay of Bengal, 483-488

Bayesian Analysis, 119-124, 355-364, 373-380,

1109-1114

Bayesian Method, 1109-1114

Bayesian Statistics, 381-390

Beach Rock, 887-898

Bengal Shelf, 909-916, 917-928
Beth Sheam, 1333-1342

Biblical Studies, 127-132

Biodeposits, 637-646

Biogenic $\mathrm{CO}_{2}, 659-670$

Biomedical, 305-312

Bioturbation, 929-938

Bomb ${ }^{14} \mathrm{C}, 495-516,909-916,831-842,857-868$

Bomb-Pluse, 495-516

Bone, 249-254, 465-472, 909-916

Bronze Age Chronology, 1115-1120

Calibration, 119-124, 345-354, 355-364, 373-380,

449-452, 461-464, 1191-1202

Carbon, 283-292

Carbon Dioxide, 659-670, 695-702

Carbon Flux, 843-856

Carbonal, 299-304

Carbonate Material, 169-176

Castor Oil, 127-132

Caucasus, 629-636

Cave of the Treasure, 1247-1254

Cave Painting, 977-986

Central Europe, 1057-1063

Ceramics, 1255-1278

Chalcolithic, 1217-1222

Charcoal, 27-44, 45-54, 595-602, 977-986, 1139-1146

Charred Residue, 1129-1138

Chitin, 199-208

Chronological Order, 373-380

Chronology, 83-86, 1147-1154, 1255-1278,

1321-1332, 1343-1352

Clay-Bound Carbon, 255-262

Client-Server, 325-338

Climate, 15-26, 1167-1178

$\mathrm{CO}_{2}$ Trapping, 293-298

Coleoptera, 1007-1020

Collagen, 909-916

Comparison, 1085-1108

Compound-Specific Radiocarbon Analysis (CSRA),

209-216, 949-956

Connecticut, 391-402

Conservation, 229-238

Contamination, 127-132, 275-282

Coral, 15-26, 473-476, 899-908

Cosmic Background, 157-162

Cosmogenic Radiation, 731-742, 759-764

Cosmogenic Isotopes, 365-372, 743-750

Cremation, 249-254

Cross-Dating, 417-424

Cyclones, 917-928

Danube, 465-472

Data Model, 325-338

Databases, 325-338

Dating (see also Radiocarbon and AMS), 69-76,

141-148, 191-198, 637-646, 773-782, 977-986 
Dead Sea, 1179-1190, 1371-1382, 1383-1390

Dead Sea Decontamination of Charcoal, 1139-1146

Dead Sea Scrolls, 127-132

Delta $(\Delta) \mathrm{R}, 461-464,483-488$

Dendrochronology (see also Tree Rings), 403-416, 417-424, 439-448

Diagenesis, 773-782

Diapir Uplift, 1179-1190

Diet, 7-14, 711-722

Diffusion, 773-782

Dinghusam, 671-678

Dissolved Organic Carbon, 183-190

Early Bronze Age,1321-1332

East Asian Monsoon, 619-628

Ecology, 7-14

Egypt, 1147-1154, 1255-1278, 1321-1332

Elevated $\mathrm{CO}_{2}, 691-694$

End-Mehiber Modeling, 939-948

Environment, 45-54, 679-690, 1147-1154

Environment Monitor, 695-702

Enzymatic, 209-216

Estonia, 809-820

Europe, 1085-1108

Evolution, 603-610

Exposure Dating, 759-764

Extinction, 69-76

Extraction System, 263-270

Fan Deltas, 1383-1390

Fatty Acids, 949-956

Fire History, 27-44

Fluorescence, 125-126

Foraminipera, 929-938

Forest Soil, 671-678

Formation Processes, 997-986

Fossil Bones, 1021-1028

Fossil Fuel, 695-702

Gedus Morhua, 843-856

Gamma Spectrometry, 917-928

Gas Ion Source, 149-156

Geochronology, 325-338

Geomorphology, 731-742

Geothermal, 7-14

Ghassulian,1217-1222

Glaciology, 731-742

Global Carbon Cycle, 743-750

Grain-Size Distribution, 939-948

Graphite, 293-298

Greece, 1029-1048

Greenland, 495-516

Groundwater, 183-190, 773-782

Hard-Water Effect Variations, 821-830

Hemisphere Offset, 119-124

Herforth, 125-126
High pH Anion Exchange Chromatography, 209-216

Historic Earthquakes, 1371-1382, 1383-1390

Hokkaido, 465-472

Holocene, 69-76, 571-580, 647-658, 703-710,

887-898, 899-909

Hula Basin, 561-570

Human Fraction, 595-602

Human Impact, 619-628

Human Occupation of Australia, 1139-1146

Humus, 751-758

HVEE, 149-156

Hvon Pine, 449-452

Hydrogeochemistry, 793-800

Iceberg Discharge, 939-948

Icons, 1064-1076

Inbuilt Age, 27-44

Incipient Pottery Insect, 199-208

Inter-Comparison, 321-325, 1064-1076

Inversion, 603-610

Ireland, 517-526

Irish Sea, $869-878$

Iron, 221-228

Iron Age, 1343-1352

Iron Gates Gorge, 453-460

Iskendrun Bay, 957-964

Isotopes, 15-26, 275-282, 603-610, 647-658, 887-898

Isotope Hydrology, 325-338

Israel, 1241-1254, 1333-1342

Italy, 489-495, 1049-1056

Japan, 899-908

Japanese Cypress, 433-428

Jericho, 1321-1332

Jordan, 1217-1222

Kaistic Aquifers, 793-800

Kallmann, 125-126

Kennewick Skeleton, 965-976

Kiev, 1064-1076

Korean Peninsula, 555-560

Kurgan, 629-636

Kyushu Island, 703-710

Laboratory Protocols, 325-338

Lake ${ }^{14} \mathrm{C}$ Budget, 821-830

Lake Atmosphere ${ }^{14} \mathrm{C}$ Relationship, 821-830

Lake Gosciaz, 831-842

Lake Kinneret ${ }^{14} \mathrm{C}, 821-830$

Lake Levels, 1179-1190

Lake Sediments, 831-842

Lake Vegetation, 809-820

Last Glacial Period, 433-438

Late-Glacial, 997-1006, 339-344

Late Pleistocene, 1167-1178

Late Prehistory, 1203-1216

Late Quaternary, 45-54 
Leaves, 695-702

Levant, 1333-1342

Lichen, 637-646

Lipid, 191-198

Liquid Scintillation Counting, 125-126, 157-162, 695-702

Liquification, 1371-1382

Load Structure Deformations, 1383-1390

Loess 611-618

Log Boats, 403-416

Ma'rib, 1363-1370

Macrofossils, plant, 1007-1020

Marine Reservoir Effect, 465-472

Marine Sediment, 929-938, 949-956

Mathematical Modeling, 345-354

Mediterranean Pottery, 1343-1352

Megafauna, 69-76

Mercury, 495-516

Mercury Atmosphere Deposition, 801-808

Mesolithic, 997-1006, 1029-1048

Meteorite, 263-270

Meterology, 659-702

Middle East,1191-1202

Mitrochondrial DNA, 965-976

Modeling the Time Series, 365-372

Monte Carlo, 339-344

Mortar, 271-274

Mount Sedom, 1179-1190

Mueti Aquifer System, 793-800

Muroscale ${ }^{14} \mathrm{C}$ Analysis, 949-956

Nagaed-Der, 1255-1278

Neanderthals, 1021-1028

Near East, 1147-1154, 1155-1166, 1191-1202

Negev, 1203-1216

Neural Non-Linear Approach, 365-372

Neolithic,1191-1202

Noah's Flood, 1247-1254

North Pacific, 465-472, 857-868, 949-956

Northwest Pacific, 473-476, 477-482

Nuclear Fuel Cycle, 869-878

Occupation Phases, 887-898

Ocean Circulation, 939-948

Organic Carbon, 555-560

Organic Matter, 611-618

Otoliths, 843-856

Oxalates, 637-646

Oxcel, 355-364

Pacific (North and Northwest) 465-472, 473-476, 477-482, 857-868, 949-956,

Pacific Ocean, 879-886

Palaeodiet, 453-460

Paleoamerican, 965-976

Paleoclimate, 439-448, 647-658
Paleoecological Analysis, 703-710

Paleolithic, 977-986, 1029-1048

Paleosol, 177-182, 629-636

Peat, 391-402, 495-516, 517-526, 561-570

Peat Bogs, 571-580

Performance, 149-156

Polymer Hydrolysis, 209-216

Porewater, 773-782

Palynological Sequence, 561-570

Parameters 773-782

Phoenicia, 1343-1352

Pottery, 191-198

Precision, 321-324

Predynastic, 1255-1278

Preliminary, 997-1006

Pre-Nuclear ${ }^{14} \mathrm{C}, 483-488$

Presample Age, 27-44

Preservation, 1167-1178

Prior Probability, 373-380

Probability Distribution, 1371-1382

Procedural Blanks, 275-282

Quality Control, 163-168, 325-338, 1155-1166

Quantitative Analysis, 365-372

Quaternary, 561-570

Qumran, 127-132

Radioactive and Stable Isotopes, 793-800

Radioactive Waste, 879-886

Radiocaesium, 831-842

Radiocarbon (see also ${ }^{14} \mathrm{C}$ ), 141-148, 163-168, 191198, 209-216, 221-228, 229-238, 271-274, 275-282, 299-304, 305-312, 313-320, 403-416, 417-424, 439448, 461-464, 517-526, 603-610, 611-618, 671-678, 731-742, 743-750, 831-842, 843-856, 879-886, 929938, 987-996, 1029-1048, 1085-1108, 1139-1146, 1167-1178

Radiocarbon Age, 887-898, 957-964

Radiocarbon in Seawater, 857-868

Radiometric Dates, 1155-1166, 1343-1352

Rattus exulan, 711-722

Rattus norvegicus, 7-14

Reaction Rate, 271-274

Recover Missing Data, 365-372

Reedmat, 1247-1254

Regional Correlation, 1179-1190

Rejuvenation, 603-610

Reliability, 321-324

Reservoir Age, 7-14, 119-124, 453-460, 461-464, 473-477, 477-482, 483-488, 489-494

Review, 141-148

Rotoehu Ash, 239-248

Rust Methods, 221-228

Ryukyu Islands, 899-908

Sabir Culture, 1353-1362

Salt Marsh, 391-402 
Sample Preparation and Selection, 163-168, 169-176,

177-182, 183-190, 611-618, 1007-1020

Scythian Sites and Cultures, 417-424, 425-432, 1085-1108

Sea of Japan, 477-482

Sea-Level Change, 391-402, 887-898

Sea-Surface Temperature, $15-26$

Seasonal ${ }^{13} \mathrm{C}$ Fluctuation, 433-438

Seawater, 773-782, 879-886

Seburi Mountains, 703-710

Sediment and Soils, 27-44, 255-262, 671-678, 821-

830, 831-842, 909-916, 917-928, 987-996, 1007-1020

Seismites, 1371-1382, 1383-1390

Sellafield, 869-878

Seoul National University, 163-168

Serbia, 647-658

Seriation, 345-354

Settlement, 987-996, 1203-1216

Shell, 83-86

Siberia, 425-432,

Sinai, $1203-1216$

SIS, 305-312

Solar Activity, 339-344, 439-448

SOM, 595-602

South America, 69-76

South Kamchatka, 571-580

Southern Jordan, 1203-1216

Southern Levant, 1217-1222

Southern Siberia, 417-424

Spectral Analysis, 417-424

Speleothems, 647-658

Stable Isotopes, 199-208

Statistical Presentation, 355-364, 1191-1202

Steppe Zone (Russian), 1115-1120

Stepped-Combustion, 255-262

Stone Tools,759-764

Stratigraphy, 345-354, 917-928

Stuiver and Polach, 691-694

Sub-Fossil, 199-208

Submerged Site, 1167-1178

Sub-Milligram ${ }^{14} \mathrm{C}$ Samples, 275-282

Subsistence Technology, 83-86
Suess Effect, 679-690

Swamp, 555-560

Tandetron, 149-156

Target Preparation, 275-282, 283-292

Tectonic Uplift, 957-964

Tel Dor, 1343-1352

Tel Rehov, 1333-1342

Temple of Bar'an, 1363-1370

Terminal Chalcolithic, 1247-1254

Terrestrial Age, 263-270

Theopetra Cave, 1029-1048

Thessaly, 1029-1048

Tianma-Qucun Site, 1109-1114

Tiri Whalebone, 711-722

Tree Rings (see also Dendrochronology), 433-438, 449-452, 679-690

Tritium, 773-782, 783-792

Trophic Levels, 199-208

Turkey, 957-964

Typo-Chronology, 997-1006

U/Th Dating, 561-570

Underground Laboratory, 157-162

Upper Volga Sites, 571-580

Urban Forest, 659-670

Variable Influence of Source Activity, 809-820

Velzeke, 987-996

Vertisol, 603-610

Viandija Cave, 1021-1028

Volcanic Eruptions, 571-580

Water Levels, 1167-1178

Western U.S., 965-976

Wiggle-Matching, 391-402, 425-432

Wiggle-Matching

Windscale, 517-526

Wood, 177-182

Yemen, 1353-1362, 1363-1370

Young Neolithics, 1057-1063 